R. Carlsson

Nagoya Math. J.

Vol. 78 (1980), 45-56

\title{
$N$-ARY ALGEBRAS
}

\author{
RENATE CARLSSON
}

\section{Introduction}

$N$-ary algebras are modules with a $n$-fold multiplication which we assume to be associative if nothing else is stated. They are a canonical generalization of binary and ternary associative algebras. Ternary rings were first investigated by Lister [8]. The aim of this note is to show that the Wedderburn structure theory and the usual cohomology for binary associative algebras can be extended to $n$-ary algebras. For ternary algebras this has been done in [8] and [1]. Moreover analogous results are wellknown for Lie and alternative triple systems, and for ternary Jordan pairs.

$N$-ary algebras are a special case of algebras with $n$-ary multiplication operators which were studied especially by Kurosh [7]. Let $A$ be a not necessarily associative $n$-ary algebra over a ring $k$. Assume that the $k$-module $A$ is free over $k$ of finite rank $r$. Then $A$ corresponds to a system of $r$ differential equations in $r$ variables $x_{i}, i=1, \cdots, r, \dot{x}_{j}=p_{j}(x)$, $x:=\left(x_{j}\right)_{j=1}^{r}, p_{j}(x) \in k[x], p_{j}$ a homogeneous polynomial of degree $n$, shortly $\dot{x}=p(x), p$ a homogeneous vector polynomial. This has been established by Röhrl [11].

So one notes that the result analogous to the Wedderburn decomposition as shown in the following implies the complete reducibility of the corresponding system of differential equations.

The proofs in the subsequent make use of the theory for binary and ternary associative algebras.

\section{Definitions and imbeddings}

Let $k$ denote a commutative and associative ring with a unit 1 . The $k$-modules $U$ considered are taken to be unitary. Let $U^{[n]}$ for $n \in N$ denote the $n$-fold cartesian product. Then a $n$-multiple system $A$ for

Received May 20, 1978. 
$n \in N \backslash\{1\}$ is a $k$-module together with a $n$-linear inner composition $\mu: A^{[n]}$ $\rightarrow A$ with

$$
\left(x_{i}\right)_{i=1}^{n}:=\left(x_{i}\right)_{i=1, \cdots, n} \mapsto\left\langle x_{i}\right\rangle_{i=1}^{n}
$$

where $x_{i} \in A$. The multiple system is called associative or in short a $n$ ary algebra if the following associativity conditions are valid. Let $y_{i} \in A$ with $y_{1}:=\left\langle x_{i}\right\rangle_{i=1}^{n}$, the $x_{i}$ as afore. Then for any $r \in\{2, \cdots, n\}$

$$
\left\langle y_{i}\right\rangle_{i=1}^{n}=\left\langle z_{i}\right\rangle_{i=1}^{n}
$$

with $z_{i}:=x_{i}$ when $1 \leq i<r, z_{i}:=y_{i}$ for $r<i \leq n$, and $z_{r}:=\left\langle v_{i}\right\rangle_{i=1}^{n}$ with $v_{i}:=x_{r-1+i}$ if $1 \leq i \leq i_{0}:=n-r+1$ and $v_{i_{0}+j}:=y_{1+j}$ if $1 \leq j<r$. More informally, for any $r$

$$
\mu\left(\mu\left(x_{1}, \cdots, x_{n}\right), y_{2}, \cdots, y_{n}\right)=\mu\left(x_{1}, \cdots, x_{r-1}, \mu\left(x_{r}, \cdots, y_{r}\right), y_{r+1}, \cdots, y_{n}\right) .
$$

By (1) the multiplication in $A$ is associative in the obvious sense. The length of any monomial is congruent to 1 modulo $n-1$. If $n=2$ then $A$ is a binary associative algebra.

For an associative $k$-algebra $B, x_{i} \in B$, and $s \in N$, let $\prod_{i=1}^{1} x_{i}:=x_{1}$ further $\prod_{i=1}^{s+1} x_{i}:=\left(\prod_{i=1}^{s} x_{i}\right) \cdot x_{s+1}$. Suppose that $A$ is a $r$-ary algebra, and $x_{i} \in A$ for $i \in\{0, \cdots, r-1\}$. Define the right multiplication $\rho$ by

$$
\rho\left(x_{i}\right)_{i=1}^{r-1}: x_{0} \mapsto\left\langle x_{i}\right\rangle_{i=0}^{r-1} .
$$

If $d \in N \backslash\{1\}, n:=(r-1) \cdot(d-1)+1$ then we consider $A$ as a $n$-ary algebra $A_{\tau(d)}$ by

$$
\left\langle x_{i}\right\rangle_{i=0}^{n-1}:=\left(\prod_{j=0}^{d-2} \rho\left(x_{j(r-1)+i}\right)_{i=1}^{r-1}\right)\left(x_{0}\right) .
$$

$A_{\tau(d)}$ is a $d$-ary algebra if $r=2$. Define $A^{1}:=A$ and

$$
A^{s(r-1)+1}:=\left(\prod_{j=1}^{s} \rho\left(A_{i j}\right)_{i=1}^{r-1}\right)(A) \quad \text { where } A_{i j}:=A \text { and } s \in N .
$$

For a $n$-ary algebra $A$ the concepts of a (n-ary) morphism, automorphism, ideal, subalgebra etc. are defined obviously (cf. [2]). For a submodule $B$ we denote by ${ }^{(1)} B$ the $k$-module generated by the monomials $\left\langle x_{i}\right\rangle_{i=1}^{n}$ with $x_{i} \in A$ and $x_{i_{1}}, x_{i_{2}} \in B$ for two different subscripts. If $B$ is an ideal in $A$ then obviously ${ }^{(1)} B$ is an ideal.

Examples. 1.) Let $A$ be a ternary ring.

2). For $n \in N, n \geq 2, Z_{n-1}$ the rational integers modulo $n-1$, and 
$i \in Z_{n-1}$ let $V_{i}$ denote a $k$-module. Set $F_{i}:=\operatorname{Mor}_{k}\left(V_{i}, V_{i+1}\right)$, the $k$-module of the module morphisms, and $F:=\oplus F_{i}$ for $i \in Z_{n-1}$. The structure of a $n$-ary algebra can be induced on $F$ obviously as follows: If $f_{i} \in F_{i}$ and if $f_{j+1} f_{j}$ for $j \in Z_{n-1}$ denotes the product of maps then with $s_{t} \in Z_{n-1}$ let

$$
\left\langle f_{s_{t}}\right\rangle_{t=1}^{n}:=\left\{\begin{array}{cl}
\prod_{t=1}^{n} f_{s_{t}} & \text { if } s_{t-1}=s_{t}+1 \text { when } t>1 . \\
0 & \text { otherwise }
\end{array}\right.
$$

$\Pi$ defined obviously. If any $V_{i}$ is free of finite rank $r_{i}>0$ we denote this $n$-ary algebra by $k\left(r_{i}\right)_{i=1}^{n-1}$.

3.) If $A$ is an associative algebra, $\varphi$ an automorphism with $\varphi^{n-1}=$ $\mathrm{Id}_{A}$, and $\xi \in k, \xi$ a $n-1$ th root of unity then the corresponding rootspace is a $n$-ary subalgebra of $A_{\tau(n)}$.

Define a $n$-multiple module over $A$ to be a $k$-module $M$ together with $n$ multilinear maps $\mu_{j}$,

$$
\mu_{j}: A^{[n-1]} \times M \rightarrow M
$$

where $j \in\{1, \cdots, n\}$. The $k$-module direct sum $E_{0}:=A \oplus M$ can be considered as a $n$-multiple system with ${ }^{(1)} M:=\{0\}$, the $n$-linear multiplication in $E_{0}$ extending $\mu$ and the $\mu_{j} . \quad M$ is called a (associative) n-ary module over $A$ if $E_{0}$ is associative. $E_{0}$ is called the semidirect sum of $A$ and $M$ or the split zero extension of $A$ by $M$. In the following we denote by $A$ a $n$-ary algebra and by $M$ a $n$-ary module over $A$ if we state nothing different.

Similarly as in the ternary case we can imbed $A$ into an associative algebra $S(A)$. For this let $E(A):=\oplus_{j=1}^{n-1} \otimes{ }^{j} A$. We induce the structure of a binary algebra on the $k$-module $E(A)$ as follows. If $p, q \in\{1, \cdots, n-1\}$ let $x:=\bigotimes_{i=1}^{p} x_{i}, y:=\bigotimes_{j=1}^{q} y_{j}$ with $x_{i}, y_{j} \in A$. Then

$$
x \circ y:= \begin{cases}x \otimes y & \text { if } p+q \leq n-1 \\ \bigotimes_{j=1}^{p+q-(n-1)} z_{j} & \text { if } p+q \geq n .\end{cases}
$$

For the latter case let $u_{i}:=x_{i}$ when $1 \leq i \leq p, u_{i}:=y_{i-p}$ if $p<i \leq n$, further $z_{1}:=\left\langle u_{i}\right\rangle_{i=1}^{n}$, and $z_{j}:=y_{j+(n-p)}$ otherwise. Then any product of length not exceeding $n$ is associative.

Let $K$ denote the submodule of $E(A)$ generated by

$$
\begin{gathered}
\left\{v \mid \exists j, 2 \leq j \leq n-1 \forall m_{1}, m_{2} \in N, m_{1}+m_{2}=n-j: v \in A^{j}:=\otimes{ }^{j} A\right. \\
\left.\wedge v \circ A^{n-j}=A^{n-j} \circ v=A^{m_{1}} \circ v \circ A^{m_{2}}=\{0\}\right\} .
\end{gathered}
$$


It is shown by an obvious verification that $K$ is an ideal in $E(A)$, and that $S(A):=E(A) / K$ is an associative algebra. Then $\iota: A \rightarrow S(A)$ with $a \mapsto a+K$ is a $n$-ary monomorphism of $A$ into $S(A)_{\tau(n)}$. We call $S(A)$ the standard imbedding of $A$.

Let $B$ denote an associative algebra with multiplication $(y, z) \mapsto y \circ z$ for $y, z \in B$, together with a map $\iota: A \rightarrow B$. Then $(B, \iota)$ is called an imbedding of $A$ if $\iota$ is a monomorphism of $A$ in $B_{\tau(n)}$, and $B=\sum_{j=1}^{n-1} \iota(B)^{j}$. Then $\prod_{i=1}^{n} \iota\left(x_{i}\right)=\iota\left(\left\langle x_{i}\right\rangle_{i=1}^{n}\right)$ for $x_{i} \in A$, the product taken in $B$. If the sum is direct then the imbedding is direct. For a direct imbedding let $\varepsilon_{j}: B$ $\rightarrow B_{j}:=(\iota(A))^{j}$ be the canonical projection. Then $B_{i} \circ B_{j}=B_{p}$ with $p=$ $i+j$ modulo $(n-1), p \in\{1, \cdots, n-1\}$. If $\xi \in k, \xi$ a primitive $n-1$ th root of unity, $w \in B$ and $w=\sum_{j=1}^{n-1} w_{j}$ with $w_{j} \in B_{j}$ then $\varphi: B \rightarrow B$ with $w \mapsto \sum_{j=1}^{n-1} \xi^{j} w_{j}$ is an automorphism of $B$. An imbedding $(B, \iota)$ of $A$ is called universal if for any imbedding $(C, \kappa)$ of $A$ there exists a (unique) morphism $f: B \rightarrow C$ of the algebras with $f_{\iota}=\kappa$.

Proposition 1. Let $A$ denote an associative $n$-ary algebra over $k$. Then there exists a universal imbedding $(U(A), \iota)$ of $A .(U(A), \iota)$ is direct.

Proof. Let $T^{*}(A):=\bigoplus_{n \in N}\left(\otimes^{n} A\right)$ denote the nonunitary tensor algebra of the $k$-module $A$. The ideal $Q$ of $T^{*}(A)$ is generated by $\otimes_{i=1}^{n} x_{i}$ $-\left\langle x_{i}\right\rangle_{i=1}^{n}$ for $x_{i} \in A$. Set $U(A):=T^{*}(A) / Q$, further $\iota: x \mapsto x+Q$. Then $(U(A), \iota)$ is an imbedding by the existence of the standard imbedding, and the elementary properties of the tensor product. From the latter the imbedding is direct.

Note that $U(A) \cong \bigoplus_{j=1}^{n-1}\left(\otimes^{j} A\right)$. In the following we may suppose that $\iota$ is the inclusion map. Define the subset $V$ of $U(A)$ by substituting $U(A)$ instead of $E(A)$ in (2). Let $J(A)$ denote the ideal spanned by $V$. Obviously $S(A) \cong U(A) / J(A)$.

\section{Radical and semisimplicity}

Let $A$ be a $n$-ary algebra. We define the Jacobson radical $R(A)$ or $R$ of $A$. If $n=2$ let [9]

$$
R(A):=\{x \in A \mid \forall y \in A \exists z \in A: x+z=x \cdot y \cdot z=z \cdot y \cdot x\} .
$$

If $n>2$ set $R(A):=R(U(A)) \cap A . \quad R(A)$ is an ideal of $A . A$ is called semisimple if $R(A)=\{0\}$.

For $r \in N_{0}$ let $A_{(r)}:=A^{r(n-1)+1}$. We call $A$ nilpotent if $A_{(r)}=\{0\}$ for some $r$. For an ideal $I$ set ${ }^{(0)} I:=I$ and ${ }^{(r+1)} I:={ }^{(1)}\left({ }^{(r)} I\right)$ if $r \in N$. Then 
the ${ }^{(r)} I$ define a descending chain of ideals in $A . \quad I$ is called $A$-solvable if ${ }^{(r)} I=\{0\}$ for some $r \in N_{0}$. Obviously the sum of $A$-solvable ideals is $A$ solvable.

Lemma 1. An ideal I in a n-ary algebra is nilpotent if and only if $I$ is A-solvable.

Proof. If $r \in N_{0}$ define $d_{r} \in N_{0}$ by $n^{r}=d_{r} \cdot(n-1)+1$. Since ${ }^{(r)} I \supset I_{\left(d_{r}\right)}$, $A$-solvability implies nilpotency.

On the other hand the defining monomials $z$ in ${ }^{(r)} I$ have $2^{r}$ factors in $I$. Let $r=2 n$. We may assume that the product of any $n$ consecutive factors in each $z$ is in $I$. Now $2^{2 n} / n \geq 2 n-1$. Thus

$$
{ }^{(2 n)} I \subset I_{(2)} \text {. }
$$

Assume ${ }^{(2 s n)} I \subset I_{(2 s)}$ for some $s \in N$. Then

$$
\left.{ }^{(2(s+1) n)} I \subset{ }^{(2 s n)}{ }^{(2 n)} I\right) \subset\left(^{(2 n)} I\right)_{(2 s)} \subset\left(I_{(2)}\right)_{(2 s)} \subset I_{(2(s+1))} .
$$

Hence nilpotency implies $A$-solvability.

LEMMA 2. Any nilpotent ideal $I$ in a n-ary algebra $A$ generates a nilpotent ideal in $U(A)$.

Proof. For an ideal $I$ in $A$ denote by $I[A]$ the ideal in $U(A)$ generated by $I$. If $r \in N_{0}$ hence

$$
(I[A])^{n r} \subset\left({ }^{(r)} I\right)[A] .
$$

Thus by Lemma 1 if $I$ is nilpotent then $I[A]$ is nilpotent.

We call $A$ artinian if $U(A)$ is an artinian ring. By Hopkins Theorem $R(U(A))$ is nilpotent. Hence

CoRollary 1. The radical $R$ of an artinian n-ary algebra $A$ is the maximal nilpotent ideal in $A$.

If the ring $k$ contains a primitive $n-1$ th root of unity then $k$ is called $n-1$ split. Let $k$ and $\tilde{k}$ be fields with $k \subset \tilde{k}$ : The base field extension $A_{\tilde{k}}$ : $=A \otimes_{k} \tilde{k}$ can be considered as a $n$-ary algebra in the obvious way. Call $A$ separable if $A_{\tilde{k}}$ is semisimple for any $\tilde{k}$. By standard properties of scalar extensions one derives $S\left(A_{\tilde{k}}\right) \cong S(A)_{\tilde{k}}$ with canonical algebra isomorphism. If $A$ is artinian then $(R(S(A)))_{\tilde{k}}$ corresponds to a submodule of $R\left(S\left(A_{\tilde{k}}\right)\right)$. 
TheOREM 1. Let $A$ be a n-ary algebra over a ring $k$.

(1) If $A$ is semisimple and $k n-1$ split, or $k$ a field with char $(k)$ $\nmid n-1$ and $A$ artinian and separable over the field then $S(A)$ is semisimple. If in the latter case $A$ is finite dimensional over $k$ then $S(A) \cong$ $U(A)$.

(2) Any semisimple direct imbedding of $A$ is isomorphic to $S(A)$.

Proof. (1) We may assume that $k$ contains a primitive $n-1$ th root of unity $\xi$, and that $A$ is semisimple. Generate the automorphism $\varphi$ of $U(A)$ by $\xi$. We consider the radical of $U(A), \tilde{R}:=R(U(A)), \quad R$ is $\varphi$-invariant by (3). Since $\varphi^{n-1}=\mathrm{Id}_{U(A)}$ then similarly as in the finite dimensional case there is a rootspace decomposition of $\tilde{R}$ for $\varphi, \tilde{R}=\oplus_{i=1}^{n-1} \tilde{R}_{i}, \xi^{i}$ the root belonging to $\tilde{R}_{i}$. Assume $m_{1}, m_{2} \in N$ and $m_{1}+m_{2}=n-i$. Since $A^{m_{1}} \circ \tilde{R}_{i} \circ A^{m_{2}}=A^{n-i} \circ \tilde{R}_{i}=\tilde{R}_{i} \circ A^{n-i}=\{0\}$ if $i \geq 2$, hence $\tilde{R} \subset J(A)$. Now $J(A)$ is nilpotent thus $J(A)=\tilde{R}$. Hence $S(A)$ is semisimple.

Let $A$ be finite dimensional over the field $k$ and $A$ separable. Then there exists a Wedderburn decomposition $U(A)=B \oplus J(A)$ of $U(A)$ with a semisimple subalgebra $B$ where $\varphi(B)=B[11]$. Since $(J(A))_{1}=\{0\}$ one has $A \subset B$, thus $U(A)=B$. Hence $J(A)=\{0\}$.

(2) Let $B$ denote an arbitrary direct and semisimple imbedding of $A$, and $f: U(A) \rightarrow B$ the corresponding morphism. If $z \in \operatorname{ker}(f)$ then $z_{i}:=\varepsilon_{i}(z) \in \operatorname{ker}(f)$ for $i=1, \cdots, n-1$. Now $z_{1}=0$. Hence with $m_{1}$ and $m_{2}$ as before then $A^{m_{1}} \circ z_{i} \circ A^{m_{2}}=A^{n-i} \circ z_{i}=z_{i} \circ A^{n-i}=0$ if $i \geq 2$. Since $J(A)$ is nilpotent $\operatorname{ker}(f) \subset J(A) \subset \operatorname{ker}(f)$. Hence $S(A) \cong B$.

We call a $n$-ary algebra $A$ with $A^{n} \neq\{0\}$ simply if $\{0\}$ and $A$ are the only ideals in $A$. We then get as a corollary

Theorem 2. Let $A$ be an artinian n-ary algebra over a ring $k, k n-1$ split, and $A$ semisimple. Then

$$
A=\bigoplus_{i=1}^{r} S_{i}, \quad r \in N_{0}
$$

where each $S_{i}$ is a simple ideal in $A$.

Proof. Let $A \neq\{0\}$. By Theorem 1 the standard imbedding $S(A)$ is semisimple. Thus $S(A)$ decomposes into a direct sum of $\varphi$-simple ideals $C_{j}, S(A)=\oplus_{j=1}^{r} C_{j}, r \in N$. Observing Theorem 1 then $A=\oplus_{j=1}^{r}\left(C_{j}\right)_{1}$ is a decomposition into a direct sum of simple ideals.

Let $M$ be a $n$-ary module over $A$ and $E_{0}=A \oplus M$ the semidirect 
sum. If $M_{S}$ denotes the ideal in $S\left(E_{0}\right)$ generated by $M$ then $M_{S}$ is a bimodule for $U(A)$ in the obvious way. $M_{S}$ is called the standard imbedding of $M$. Irreducible $n$-ary modules over $A$ and complete reducibility are defined obviously. We then have further

Proposition 2. Suppose that $k$ is a $n-1$ split field, and $M a n$-ary module over a semisimple n-ary k-algebra $A$, the dimensions finite. For the center $Z$ of $U(A)$ let $Z \otimes_{k} Z$ be semisimple. Then $M$ is completely reducible over $A$.

Proof. Let $M \neq\{0\}$ and $W:=U(A) \otimes_{k} U(A)^{\text {op }}$. Then $M_{S}$ can be considered as an associative left module over $W$ where for $a, b \in U(A)$ and $m \in M_{S}$ one has $(a \otimes b) m=a \circ m \circ b$. Now $M_{S}$ has a two sided $\varphi$ invariant Peirce decomposition for the unit $e \in U(A)$. Let

$$
M_{S}=\left(M_{S}\right)_{11} \oplus\left(M_{S}\right)_{10} \oplus\left(M_{S}\right)_{01} \oplus\left(M_{S}\right)_{00}
$$

be the decomposition over e. Now observe [5, p. 117] and [1], Lemma 2]. Any Peirce component is completely reducible over $W$ and over $U(A)$ from the right and from the left. Thus $M_{S}=\oplus_{i=1}^{r} N(i), r \in N$, any $N(i)$ a $\varphi$-invariant minimal subbimodule not zero for $U(A)$. Hence $M=\bigoplus_{i=1}^{r}(N(i))_{1}$ is a decomposition into irreducible $n$-ary submodules.

\section{The cohomology}

Let $B$ and $B^{\prime}$ be direct imbeddings of the $n$-ary $k$-algebras $A$ and $A^{\prime}, V$ a submodule of $B$ and $f: V^{[r]} \rightarrow B^{\prime}$ a multilinear map. Let $I:=$ $\{1, \cdots, n-1\}$. Similarly as in [1] $f$ is isovarying if $x:=\left(x_{i}\right)_{i=1}^{r} \in V^{[r]}$ with $x_{i} \in B_{j(i)}$ for $j(i) \in I$ and $j \in I$ with $j:=\sum_{i=1}^{r} j(i)$ modulo $(n-1)$ implies $f(x) \in B_{j}^{\prime} . \quad f$ is called antivarying if $f(x) \in \oplus B_{p}^{\prime}$ for $p \in I \backslash\{j\}$. Any multilinear map $f$ has a unique decomposition $f=f_{1}+f_{0}, f_{1}$ isovarying and $f_{0}$ antivarying. Let $\varepsilon: f \mapsto f_{1}$. Hence $\varepsilon^{2}:=\varepsilon \cdot \varepsilon=\varepsilon$.

We consider the Hochschild cohomology of $U(A)$ for $M_{S}$. The modules of the cochain complex are $C^{0}:=M_{S}$, and the $k$-modules $C^{q}$ of the $q$-linear maps from $U(A)^{[q]}$ in $M_{S}$ if $q \in N$. If $r \in N_{0}$ the coboundary operator $\delta: C^{r} \rightarrow C^{r+1}$ is defined for $a_{i} \in U(A)$ by

$$
\begin{aligned}
\delta f\left(a_{1}\right)= & a_{1} \cdot f-f \cdot a_{1} \quad \text { if } f \in C^{0} \\
\delta f\left(\left(a_{i}\right)_{i=1}^{q+1}\right)= & a_{1} \cdot f\left(\left(a_{i}\right)_{i=2}^{q+1}\right)+\sum_{i=1}^{q}(-1)^{i} f\left(\left(b_{i j}\right)_{j=1}^{q}\right) \\
& +(-1)^{q+1} f\left(\left(a_{i}\right)_{i=1}^{q}\right) \cdot a_{q+1} \quad \text { if } f \in C^{q}
\end{aligned}
$$


with $b_{i j}:=a_{j}$ if $j<i, b_{i i}:=a_{i} \circ a_{i+1}$, and $b_{i j}:=a_{j+1}$ if $i<j$. The $k$ modules of $r$-cocycles and $r$-coboundaries are denoted $Z^{r}$ and $B^{r}$ where $B^{0}:=\{0\}$, and $H^{r}\left(U(A), M_{S}\right):=Z^{r} / B^{r}$ is the $r$-dimensional cohomology group. Extend $\varepsilon$ to $C^{0}$ by $\varepsilon:=\varepsilon_{n-1}$. Any $\varepsilon$-invariant submodule $T$ of $C^{r}$ decomposes, $T=T_{1} \oplus T_{0}$ with $T_{i}:=\left\{f \in C^{r} \mid \varepsilon f=i \cdot f\right\}$ for $i \in\{0,1\}$. Let $C_{i}^{r}:=\left(C^{r}\right)_{i}$. Thus $C^{r}=C_{1}^{r} \oplus C_{0}^{r}$, and $\delta\left(C_{i}^{r}\right) \subset C_{i}^{r+1}$.

Set $C^{r}(A, M):=C_{1}^{r}$ for $r \in N_{0}$. Then the $C^{r}(A, M)$ together with the restriction $\partial$ of $\delta$ onto the $C_{1}^{r}$ define a cohomology complex of $A$ for $M$. The $r$-cocycles and $r$-coboundaries are $Z^{r}(A, M):=Z_{1}^{r}$ respectively $B^{r}(A, M)$ : $=B_{1}^{r}$. Denote the $r$-dimensional cohomology group by $H^{r}(A, M)$. Hence

Lemma 3. If $A$ is a n-ary algebra over a ring $k$ then for $r \in N_{0}$

$$
\begin{aligned}
H^{r}(A, M) & =Z_{1}^{r} / B_{1}^{r}, \quad \text { and } \\
H^{r}\left(U(A), M_{S}\right) & \cong H^{r}(A, M) \oplus Z_{0}^{r} / B_{0}^{r} .
\end{aligned}
$$

Corollary 2. Let $k$ be a field, char $(k) \nmid n-1, A$ separable and finite dimensional over $k$. Then for all $r \in N$ and all $n$-ary $A$-modules $M$

$$
H^{r}(A, M)=\{0\} .
$$

Proof. By Theorem 1, (1) $U(A)$ is separable.

A linear map $D: A \rightarrow M$ is called a derivation of $A$ in $M$ if

$$
D\left(\left\langle a_{i}\right\rangle_{i=1}^{n}\right)=\sum_{i=1}^{n}\left\langle b_{i j}\right\rangle_{j=1}^{n}
$$

with $b_{i j}:=a_{j}$ if $j \neq i$ and $b_{i i}:=D\left(a_{i}\right)$.

If $D(A, M)$ denotes the $k$-module of the derivations of $A$ in $M$ then $D(A, M) \cong Z^{1}(A, M)$, the isomorphism given by the restriction of $f \in Z^{1}(A, M)$ onto $A,\left.f\right|_{A}$. Inner derivations are the restrictions of the elements in $B^{1}(A, M)$ onto $A$. Hence

Corollary 3. If $A$ and $M$ are as in Corollary 2 then any derivation of $A$ in $M$ is inner.

A short exact sequence of $n$-ary $k$-algebras $M, E, A$, the connecting maps $e$ and $p n$-ary algebra morphisms,

$$
\{0\} \longrightarrow M \stackrel{e}{\longrightarrow} E \stackrel{p}{\longrightarrow} A \longrightarrow\{0\}
$$


is an extension of $A$ by $M$. The extension is singular if ${ }^{(1)}(e(M))=\{0\}$ in $E$. The equivalence and splitting of extensions is defined in the obvious way.

Let $M(E)$ denote the ideal in $U(E)$ generated by $e(M)$, and $P:=M(E)$ $\cap J(E)$. Set $U^{\prime}(E):=U(E) / P$.

For any singular extension there is a commutative diagram with exact rows

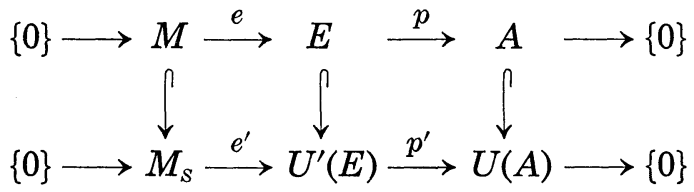

$\longrightarrow$ the inclusion map and $e^{\prime}, p^{\prime}$ algebra morphisms invariant for $\varepsilon$ (cf. [3], pp. 158-159). A $k$-module $V$ is $k$-projective if any $k$-module extension of $V$ is split. The tensor product of two projective $k$-modules is $k$ projective. Thus if $A$ is $k$-projective there exist two $k$-linear sections $q: A \rightarrow E$ and $q^{\prime}: U(A) \rightarrow U^{\prime}(E)$ with $\varepsilon q^{\prime}=q^{\prime}$ and $\left.q^{\prime}\right|_{A}=q$.

For a $n$-ary $A$-module $M$ a factor set of $A$ in $M$ is a $n$-linear map $f: A^{[n]} \rightarrow M$ so that a $n$-ary algebra $E_{f}:=A \oplus M$ is defined by

$$
\left[x_{i}\right]_{i=1}^{n}:=\left\langle x_{i}\right\rangle_{i=1}^{n}+f\left(\left(x_{i}\right)_{i=1}^{n}\right),
$$

together with ${ }^{(1)} M:=\{0\}$, and the $A$-module structure of $M . \quad f$ is trivial if there exists $\sigma \in \operatorname{Mor}_{k}(A, M)$ with

$$
f\left(\left(x_{i}\right)_{i=1}^{n}\right)=\sigma\left(\left\langle x_{i}\right\rangle_{i=1}^{n}\right)-\sum_{i=1}^{n}\left\langle b_{i j}\right\rangle_{j=1}^{n}
$$

where $b_{i j}:=x_{j}$ if $i \neq j$ and $b_{i i}:=\sigma\left(x_{i}\right)$. Let $F(A, M)$ denote the $k$-module of factor sets of $A$ in $M$, and $F_{t}(A, M)$ the submodule of trivial factor sets. Using an argument similar to the one in [3] we get

ThEOREM 3. Let $A$ be a n-ary algebra and $M$ a n-ary module over $A$, further $A$ k-projective. Then

$$
H^{2}(A, M) \cong F(A, M) / F_{t}(A, M) .
$$

There exists a 1-1 correspondence of an element in $H^{2}(A, M)$ to a class of equivalent singular extensions of $A$ by $M$. The split extensions correspond to zero. 
The proof of the statement for $A$ with radical $R$ analogous to the Wedderburn Principal Theorem reduces in the case of finite dimension over a field $k$ to ${ }^{(1)} R=\{0\}$. Hence by Corollary 2

Corollary 4. Let $k$ be a field, $A$ a finite dimensional n-ary algebra over $k, R$ the radical of $A, A / R$ separable and $\operatorname{char}(k) \nmid n-1$. Then

$$
A=S \oplus R,
$$

$S$ a semisimple n-ary subalgebra of $A$.

This corollary can also be derived directly generalizing an argument of Loos suggested for some ternary case: Since $A / R$ is separable we may suppose that $k$ is $n-1$ split. Then there is a Wedderburn decomposition

$$
U(A)=S \oplus R(U(A)),
$$

$S$ a semisimple subalgebra, and $S \varphi$-invariant by [12]. Thus $A=S_{1} \oplus R$. Obviously $S$ is a semisimple direct imbedding of $S_{1}$. By Theorem $1 S_{1}$ is semisimple.

Let $D$ be a derivation of $A$ in $A, D$ nilpotent of index $r, r \in N$, and $\operatorname{char}(k) \nmid \prod_{j=1}^{2(r-1)} j$. Then $\alpha:=\exp (D)$ is an automorphism of $A$.

For a subset $B$ of a binary associative $k$-algebra $C$ the nilindex $t$ denotes $t \in N, t$ minimal with $x^{t}=0$ for all $x \in B$ if it exists. Define $\lambda_{x}, \rho_{x} \in$ End $(C)$ by $\lambda_{x}: y \mapsto x y, \rho_{x}: y \mapsto y x$, and $\delta(x):=\lambda_{x}-\rho_{x}$. One derives the statement analogous to the Theorem of Malcev-Harish-Chandra, using an argument similar to the one in Harish-Chandra's proof for Lie algebras (cf. [5]):

Corollary 5. Let $k$ be a field, the n-ary algebra $A$ finite dimensional over $k, R$ the radical of $A$, and $A / R$ separable. Assume that $W$ and $T$ are semisimple subalgebras and $A=T \oplus R$. When $R_{s}$ denotes the ideal in $S(A)$ generated by $R$, and $t$ the nilindex of $\delta\left(R_{s}\right)_{n-1}$ for $A$ suppose $\operatorname{char}(k) \nmid(n-1) \prod_{j=1}^{2(t-1)} j$. Then there is an automorphism $\alpha$ of $A$ with $\alpha(W) \subset T$.

\section{Classification}

We determine the simple $n$-ary algebras which are finite dimensional over an algebraically closed field $k$ with $\operatorname{char}(k) \nmid n-1$. For $n=2,3$ these are wellknown. We generalize the arguments in [8] for $n=3$. 
Lemma 4. Let $k$ be a field, $A$ a n-ary algebra over $k$.

(1) If $A=k\left(r_{i}\right)_{i=1}^{n-1}, r_{i} \in N$, then $A$ is simple.

(2) Let $d, r \in N \backslash\{1\}$, and $A$ a simple r-ary algebra. Then $A_{\tau(d)}$ is simple.

Proof. (1) follows in the obvious manner using a standard basis.

For (2) assume that $A$ is a simple $r$-ary algebra and $I$ an ideal in $A_{\tau(d)}$. Let $z=\left\langle y_{i}\right\rangle_{i=1}^{r}$ with $y_{i} \in A$, and $y_{i_{0}} \in I$ for some subscript $i_{0}$. Choose $y_{i}$ with $i \neq i_{0}$. Since $y_{i} \in A=A^{(r-1)(d-2)+1}$ trivially $z \in I$. Hence $I=\{0\}$, or $I=A$.

The lemma proves one direction of

THEOREM 4. Let $k$ be an algebraically closed field, char $(k) \nmid n-1$, and $A$ a $n$-ary algebra over $k$. Then $A$ is simple if and only if

$$
A \cong\left(k\left(r_{i}\right)_{i=1}^{r-1}\right)_{\tau(d)}
$$

with $r_{i} \in N$, and $r, d \in N \backslash\{1\}$ where $n=(r-1) \cdot(d-1)+1$.

Proof. If $A$ is a simple $n$-ary algebra then by Theorem $1 U(A)$ is simple or $\varphi$-simple. The latter is evident from the proof of that theorem. Assume that $U(A)$ is simple. Then $U(A)$ is isomorphic to a full matrix algebra $k(r)$ of $r \times r$ matrices in $k, r \in N$. Since any automorphism of $U(A)$ is inner then

$$
\varphi: x \mapsto y x y^{-1}
$$

for some $y \in U(A)$. Observing $\varphi^{n-1}=\mathrm{Id}_{U(A)}$, Schur's Lemma, and the Jordan normal form we may take $y$ as a diagonal matrix. Further the diagonal of $y$ consists of $n-1$ matrices $\xi^{i} E_{r_{i}}$ for $i=1, \cdots, n-1$ and $r_{i} \in N$ where $E_{r_{i}}$ denotes the unit matrix with $r_{i}$ rows, as is shown easily. Hence $A \cong k\left(r_{i}\right)_{i=1}^{n-1}$.

Let now $U(A)$ be $\varphi$-simple but not simple. Obviously the restriction of the canonical projection $\pi$ of $U(A)$ onto a simple ideal $B$ in $U(A)$ maps $A$ monomorphically into $B_{\tau(n)}$. Let $U(A)=\oplus_{i=0}^{q-1} \varphi^{i}(B)$ with $q>1$. Then $q \mid n-1$. Set $r:=(n-1) / q+1$. Further let

$$
B_{q}:=\left\{x \in B \mid \varphi^{q}(x)=\xi^{q} x\right\} .
$$

Obviously $B_{q} \neq\{0\}$. Moreover

$$
U(A)_{1}=\left\{x \mid x:=\sum_{i=0}^{q-1} \xi^{-i} \varphi^{i}(y), y \in B_{q}\right\} .
$$


Now $B_{q}$ is a subalgebra of $B_{\tau(r)}$, and $B$ is a simple direct imbedding of the $r$-ary algebra $B_{q}$. For suitable $r_{i} \in N$ therefore $A \cong\left(k\left(r_{i}\right)_{i=1}^{r-1}\right)_{\tau(q+1)}$.

\section{REFERENCES}

[1] R. Carlsson, Cohomology of associative triple systems, Proc. Amer. Math. Soc., 60 (1976), 1-7.

[2] — Der Wedderburnsche Hauptsatz für alternative Tripelsysteme und Paare, Math. Ann., 228 (1977), 233-248.

[ 3 ] B. Harris, Cohomology of Lie triple systems and Lie-algebras with involution, Trans. Amer. Math. Soc., 98 (1961), 148-162.

[4] I. N. Herstein, Noncommutative rings, The Mathematical Association of America, Carus Mathematical Monograph, no. 15, sec. pr. 1971.

[ 5 ] N. Jacobson, Structure of rings, rev. ed. Amer. Math. Soc. Colloqu. Publ., vol. 37, Amer. Math. Soc., Providence R. I. 1964, repr. 1968.

[6] —, Lie-algebras, Interscience Tracts in Pure and Appl. Math., no. 10, Interscience, New York and London, 1962.

[7] A. G. Kurosh, Multioperator rings and algebras, Russian Math. Surveys, 24 (1969), 1-13.

[ 8 ] W. G. Lister, Ternary rings, Trans. Amer. Math. Soc., 154 (1971), 37-55.

[9] K. McCrimmon, A characterization of the Jacobson-Smiley radical, J. Algebra, 18 (1971), 565-573.

[10] F. de Meyer and E. Ingraham, Separable algebras over commutative rings, Springer, Lecture note, 181 (1971).

[11] H. Röhrl, Algebras and differential equations, Nagoya Math. J., 68 (1977), 59-122.

[12] E. J. Taft, Cleft algebras with operator groups, Portugal. Math., 20 (1961), 195-198.

Mathematisches Seminar

Universität Hamburg

Bundesstraße 55

2 Hamburg 13 